Artículo

\title{
Efecto de recubrimientos en la maduración de yaca almacenada en condición simulada de mercadeo
}

\author{
Efigenia Montalvo-González ${ }^{1}$ \\ Yolanda Nolasco-González ${ }^{2 \S}$ \\ María de Lourdes García-Magaña ${ }^{1}$ \\ Claudia M. Medellín-Bautista ${ }^{1}$ \\ Luis M. Hernández-Fuentes ${ }^{2}$ \\ Héctor González Hernández ${ }^{3}$
}

${ }^{1}$ Instituto Tecnológico de Tepic. Avenida Tecnológico núm. 2595, Lagos del Country, Tepic, Nayarit, México. CP. 63175. Tel. 311 2119400. (emontalvo@tepic.tecnm.mx; mgarcia@tepic.tecnm.mx; clmamedellinba@ittepic.edu.mx). ${ }^{2}$ Campo Experimental Santiago Ixcuintla-INIFAP. Entronque Carretera Internacional México-Nogales km 6, Santiago Ixcuintla, Nayarit, México. CP. 63300. Tel. 8000882222 , ext. 84417. (hernandez.luismartin@inifap.gob.mx). ${ }^{3}$ Colegio de Postgraduados-Campus Montecillos. Carretera México-Texcoco km 36.5, Texcoco, Estado de México. CP. 56230. Tel. 5959520200. (hgzzhdz@colpos.mx).

${ }^{\S}$ Autora para correspondencia: nolasco.yolanda@inifap.gob.mx.

\section{Resumen}

El objetivo de esta investigación fue evaluar el efecto de recubrimientos en el proceso de maduración y calidad de yaca. Los frutos en madurez fisiológica fueron tratados o no con dos recubrimientos comerciales Natural Shine ${ }^{\circledR} 505-\mathrm{OR}$ (40\% v/v, R1) o $\operatorname{Semperfresh}^{\mathrm{TM}}$ (3\% v/v, R2);

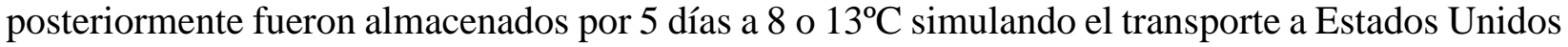
de América y después almacenados a temperatura de $25^{\circ} \mathrm{C}$, simulando su comercialización. Los frutos testigo fueron almacenados a $25^{\circ} \mathrm{C}$ hasta madurez de consumo. Se midió la velocidad de respiración (VR), producción de etileno (VPE) y parámetros físicos y químicos. Los frutos almacenados a $25^{\circ} \mathrm{C}$ presentaron el pico climatérico y máxima VPE a los 3 y 5 días de almacenamiento, respectivamente; sin embargo, los frutos sin recubrir y recubiertos almacenados en refrigeración y después a temperatura ambiente, se retrasó la aparición del pico climatérico y máxima VPE. Los frutos almacenados por 5 días a $8{ }^{\circ} \mathrm{C}$ y recubiertos con Semperfresh lograron alargar la vida de anaquel por 9 días respecto a los frutos almacenados a $25^{\circ} \mathrm{C}$ y 7 días con los frutos refrigerados a la misma temperatura, pero sin recubrir. Se concluyó que la aplicación del recubrimiento Semperfresh y el almacenamiento a $8^{\circ} \mathrm{C}$ por 5 días con posterior almacenamiento a $25^{\circ} \mathrm{C}$ puede ser una alternativa para conservar el fruto de yaca hasta por 17 días.

Palabras clave: maduración, recubrimientos, yaca.

Recibido: enero de 2021

Aceptado: marzo de 2021 


\section{Introducción}

La yaca (Artocarpus heterophyllus Lam.) es una fruta tropical de origen asiático, que ha aumentado su producción mundial a 3.7 millones de toneladas de 2015 al 2019 (FAO, 2019). En México el estado de Nayarit es el principal productor a nivel nacional con $22192.83 \mathrm{t}$ al año (SIAP, 2019). Los principales importadores de yaca mexicana son USA y Canadá (SIAP, 2019). Sin embargo, el transporte de yaca a estos destinos es por vía terrestre con una duración de 5 a 8 días entre 8 y 13 ${ }^{\circ} \mathrm{C}$, condiciones en que el fruto llega avanzado en madurez y se dispone de 2 a 3 días para comercializarlo en mercados cercanos al desembarque (Mata-Montes de Oca et al., 2007). Por lo anterior, se requiere combinar tecnologías que permitan reducir la velocidad de maduración de los frutos y prolongar su vida postcosecha, para permitir que la yaca mexicana tenga mayor margen de distribución.

Los recubrimientos para conservar frutas y hortalizas frescas hoy en día son ampliamente investigados, están compuestos de biopolímeros naturales con propiedades específicas con la función de proporcionar una barrera para disminuir la transferencia de masa (gas o líquidos) que retrasa la maduración, previenen la deshidratación, y retrasan la senescencia debido a la generación de un microclima bajo en $\mathrm{O}_{2}$ y alto $\mathrm{CO}_{2}$. Su efectividad es dependiente del cultivar, tipo de recubrimiento, y grado de madurez, principalmente (Antunes et al., 2012).

La aplicación de recubrimientos en yaca ha sido estudiada, principalmente en los bulbos del fruto y no en el fruto completo. Saxena et al. (2011) utilizaron aditivos como pretratamiento antes de aplicar quitosano en los bulbos de yaca, encontrando que el quitosano protegió los atributos sensoriales de textura y sabor que influencian en la vida útil de los bulbos precortados y que ésta fue de 46 a 48 días en atmósfera controlada (AC), de forma que las interacciones de $\mathrm{AC}$, pretratamiento y recubrimiento de quitosano en sinergia otorgan estabilidad microbiana y fisiológica al producto precortado.

Teja et al. (2016) evaluaron los efectos de diferentes recubrimientos (gel de Aloe vera y pectina) en la calidad de los bulbos de yaca durante 7 días almacenados a $6{ }^{\circ} \mathrm{C}$, concluyendo que los bulbos recubiertos con gel Aloe vera controló significativamente la pérdida de peso y ácido ascórbico, tuvo alto valor calórico, contenido proteico y vitamina $\mathrm{A}$ y $\mathrm{C}$, además que vida útil se extendió por siete días.

Vargas-Torres et al. (2017) aplicaron a bulbos de yaca sorbato de potasio/cloruro de calcio con 1metilciclopropeno (1-MCP) y aplicaron recubrimientos comestibles (goma xantana, alginato de sodio o goma gellan). Los tratamientos combinados y aplicando solo los recubrimientos mostraron disminución en la tasa de maduración y una menor pérdida de peso y respiración, en comparación el control. Además, aumento la vida útil hasta 12 días sin cambios en los atributos sensoriales, alcanzando altos valores de calidad en color, sólidos solubles totales, acidez titulable y $\mathrm{pH}$.

Natural Shine ${ }^{\circledR}$ 505-OR es un recubrimiento orgánico de alto brillo utilizado en frutos cítricos de cultivo orgánico y se recomienda para mercados nacionales de exportación. Es un producto 100\% orgánico, biodegradable aprobado bajo las regulaciones del Departamento de Agricultura de los Estados Unidos (USDA) y el Instituto de Revisión de Materiales Orgánicos (OMRI). Controla la pérdida de humedad, da brillo incluso después de la transpiración (Pace internacional, 2019). Por 
otro lado, Semperfresh ${ }^{\mathrm{TM}}$ es un recubrimiento a base de éster de sacarosa que inhibe la pérdida de agua mientras permite el intercambio de gases entre el fruto y su entorno. Reduce la pérdida de peso y el exceso de respiración, lo que resulta en cerezas más firmes con menos picaduras y sus tallos se mantienen más verdes por más tiempo, debido a una mayor retención de humedad, además da brillo sin dejar una película aceitosa o grasosa en el fruto. Su uso en pera fresca reduce las magulladuras, la pérdida de peso, preserva el color verde, sin modificar los procesos normales de maduración (Pace Internacional, 2019).

Hasta ahora, no hay reportes sobre el efecto de los recubrimientos comerciales mencionados para conservación de frutos de yaca enteros. El objetivo del estudio fue evaluar el efecto de recubrimientos comerciales en yaca almacenada en condiciones simuladas de traslado para exportación a Estados Unidos de América y almacenamiento en condiciones simuladas de mercadeo.

\section{Materiales y métodos}

En una huerta de El Llano, municipio de San Blas, Nayarit, se cosecharon frutos de yaca del material vegetal 'Agüitada' en madurez fisiológica, éstos presentaron un color de pedúnculo y cáscara verde claro ligeramente amarillento, las espinas completamente desarrolladas, separadas y con recesión, además el fruto al golpear con los dedos tuvo sonido hueco (Mata-Montes de Oca et al., 2007; Love and Paull, 2011; Luna-Esquivel et al., 2013).

Los frutos fueron lavados con agua corriente y se aplicó tiabendazol (800 ppm) como fungicida. Luego el pedúnculo fue sellado con oxicloruro de cobre (58\% p/v) (Luna-Esquivel et al., 2013). Los frutos se dividieron en siete lotes de 50 frutos cada uno, para siete tratamientos. Se emplearon tres lotes como testigo: el primero fue yaca almacenada a $25 \pm 3{ }^{\circ} \mathrm{C}$ (testigo absoluto), el segundo y tercer lote fueron: yaca almacenada a $8 \pm 2{ }^{\circ} \mathrm{C}$ ó $13 \pm 2{ }^{\circ} \mathrm{C}$ por 5 días, respectivamente, para simular el traslado a Estados Unidos y transferidos a $25 \pm 3{ }^{\circ} \mathrm{C}$ con $85-90 \%$ humedad relativa (HR) hasta su maduración para simular el mercadeo. El cuarto y quinto lote de frutos fueron tratados con recubrimientos comerciales por inmersión cuidando de cubrir completamente el fruto con la solución del recubrimiento: Natural Shine ${ }^{\circledR} 505-\mathrm{OR}$ (40\% v/v, R1), y Semperfresh ${ }^{\mathrm{TM}}$ (3\% v/v, R2,) preparados con agua, respectivamente, de acuerdo con las instrucciones del fabricante. Se dejaron secar a temperatura ambiente y posteriormente fueron almacenados en cajas de cartón a $8 \pm 2{ }^{\circ} \mathrm{C}$ por cinco días, y después a $25 \pm 3^{\circ} \mathrm{C}\left(8^{\circ} \mathrm{C} 5 \mathrm{D}+\mathrm{AMB}\right)$. A los dos lotes restantes se les aplicaron los mismos recubrimientos, pero fueron almacenados $13 \pm 2{ }^{\circ} \mathrm{C}$ por 5 días, y después a $25 \pm 3{ }^{\circ} \mathrm{C}$ $\left(13^{\circ} \mathrm{C} 5 \mathrm{D}+\mathrm{AMB}\right)$. Se midió la velocidad de respiración (VR) y velocidad de producción de etileno (VPE); pérdida fisiológica de peso (PFP), firmeza, $\mathrm{pH}$, acidez titulable (AT), sólidos solubles totales (SST), y color.

\section{Métodos de análisis}

La velocidad de respiración (VR) y velocidad de producción de etileno (VPE) se midieron por el método de Mata-Montes de Oca et al. (2007) donde frutos individuales se colocaron en recipientes herméticos (de volumen conocido) por una hora. Se tomó $1 \mathrm{ml}$ de gas del espacio de cabeza y se analizó en un cromatógrafo de gases (HP modelo 6890, USA) con una columna HP-PlotQ (15 m x $0.53 \mathrm{~mm}$ y $40 \mu \mathrm{m}$ de espesor de película), un detector de ionización de flama (FID) y uno de 
conductividad térmica (TCD). La temperatura del puerto de inyección y de los detectores fue de $250{ }^{\circ} \mathrm{C}$. Se utilizó $\mathrm{H}_{2}\left(30 \mathrm{ml} \mathrm{min}{ }^{-1}\right)$ y aire purificado $\left(400 \mathrm{ml} \mathrm{min}^{-1}\right)$. El gas acarreador fue $\mathrm{N}_{2}$ con un flujo de $7 \mathrm{ml} \mathrm{min}{ }^{-1}$. La temperatura del horno tuvo una rampa de 60 a $80{ }^{\circ} \mathrm{C}$, la cual cambió a una velocidad de $30^{\circ} \mathrm{C} \mathrm{min}^{-1}$. Se utilizó un patrón de calibración de ultra alta pureza (5.0) certificado Praxair ${ }^{\circledR}$, conteniendo $11.3 \mu \mathrm{mol} \mathrm{mol}^{-1}$ de etileno, $2.01 \mathrm{cmol} \mathrm{mol}^{-1}$ de $\mathrm{CO}_{2}, 10.1 \mathrm{cmol}$ $\mathrm{mol}^{-1}$ de $\mathrm{O}_{2}$ y $\mathrm{N}_{2}$ para balancear la mezcla.

Las concentraciones de $\mathrm{CO}_{2}$ y etileno se calcularon considerando el área y concentración del estándar, el espacio de cabeza, el tiempo de inyección, el peso y volumen del fruto. La VR se expresó en ml $\mathrm{CO}_{2} \mathrm{~kg}^{-1} \mathrm{~h}^{-1}$ y la VPE en $\mu \mathrm{kg}^{-1} \mathrm{~h}^{-1}$. La pérdida de peso (PFP) se determinó pesando los frutos diariamente con una balanza digital marca TORREY con capacidad de $20 \mathrm{~kg}$ y $0.5 \mathrm{~g}$ de precisión, la pérdida de peso se expresó como porcentaje respecto al peso inicial del fruto (Hernández-Lauzardo et al., 2007).

La firmeza de los frutos enteros y bulbos fueron determinados con un texturómetro (Stable Micro Systems, TA-XT PlusC y Reino Unido) con un puntal cilíndrico de $10 \mathrm{~mm}$ de diámetro. La firmeza de los frutos enteros se midió directamente en la parte superior, media y baja del fruto; y en nueve bulbos (sin semilla) de cada fruto. Los datos se expresaron en Newtons (N).

Los sólidos solubles totales (SST) se determinaron en jugo de pulpa fresca homogeneizada y midieron en un refractómetro (Atago, Modelo AD-13, Japón). La acidez titulable (AT) se determinó por el método volumétrico 942.15 de la AOAC (2005) en pulpa homogeneizada (5 g) con agua destilada. $(25 \mathrm{ml}$ ) y se tituló con $\mathrm{NaOH} 0.1 \mathrm{~N}$. Los resultados se expresaron en porcentaje de ácido cítrico.

El pH se midió en la pulpa homogeneizada con un potenciómetro (Jenco, Modelo 1671, Rumania). El color de la cáscara y los bulbos se midió en la parte superior, media y baja del fruto con un colorímetro Konica Minolta, CR300 (Osaka, Japón) y se reportó el color como ángulo de tono ${ }^{\circ}$ Hue.

\section{Análisis estadístico}

Se utilizó un diseño bifactorial con bloques completamente al azar, donde un factor a evaluar fue el recubrimiento, otro la temperatura de almacenamiento y los bloques los días de almacenamiento. Los datos se analizaron mediante un Anova, con comparaciones de medias por la prueba de LSD $(p \leq 0.05)$, empleando el paquete estadístico Statistica v. 10 (StatSoft, Tulsa, Oklahoma, USA).

\section{Resultados y discusión}

\section{Velocidad de respiración y producción de etileno}

El pico climatérico de la VR en los frutos testigo a $25{ }^{\circ} \mathrm{C}$ se presentó al tercer día con $108.81 \mathrm{ml}$ $\mathrm{CO}_{2} \mathrm{~kg}^{-1} \mathrm{~h}^{-1}$ (Figura 1A). En los frutos sin recubrimiento almacenados a $13{ }^{\circ} \mathrm{C}$ por cinco días y posterior simulación de mercadeo a temperatura ambiente $25^{\circ} \mathrm{C}(5 \mathrm{D}+\mathrm{AMB})$, tuvieron el pico climatérico el día 7; sin embargo, en los frutos con R1 y R2, la VR máxima se presentó a los 8 y 10 días de almacenamiento, respectivamente (Figura 1B). En los frutos sin y con recubrimiento R1 
almacenados a $8 \pm 2{ }^{\circ} \mathrm{C} 5 \mathrm{D}+\mathrm{AMB}$ (Figura 1C), la VR máxima se encontró a los 9 días y a los 14 días para frutos recubiertos con $\mathrm{R} 2$, con 179.22 y $115.85 \mathrm{ml} \mathrm{CO}_{2} \mathrm{~kg}^{-1} \mathrm{~h}^{-1}$, respectivamente. La VR aumentó drásticamente una vez que los frutos salieron de refrigeración y fueron almacenados a temperatura ambiente, esto coincide con resultados de Espinosa et al. (2013) quienes reportaron una aceleración en el metabolismo de respiración cuando frutos de guanábana se almacenaron en refrigeración y después a temperatura ambiente.
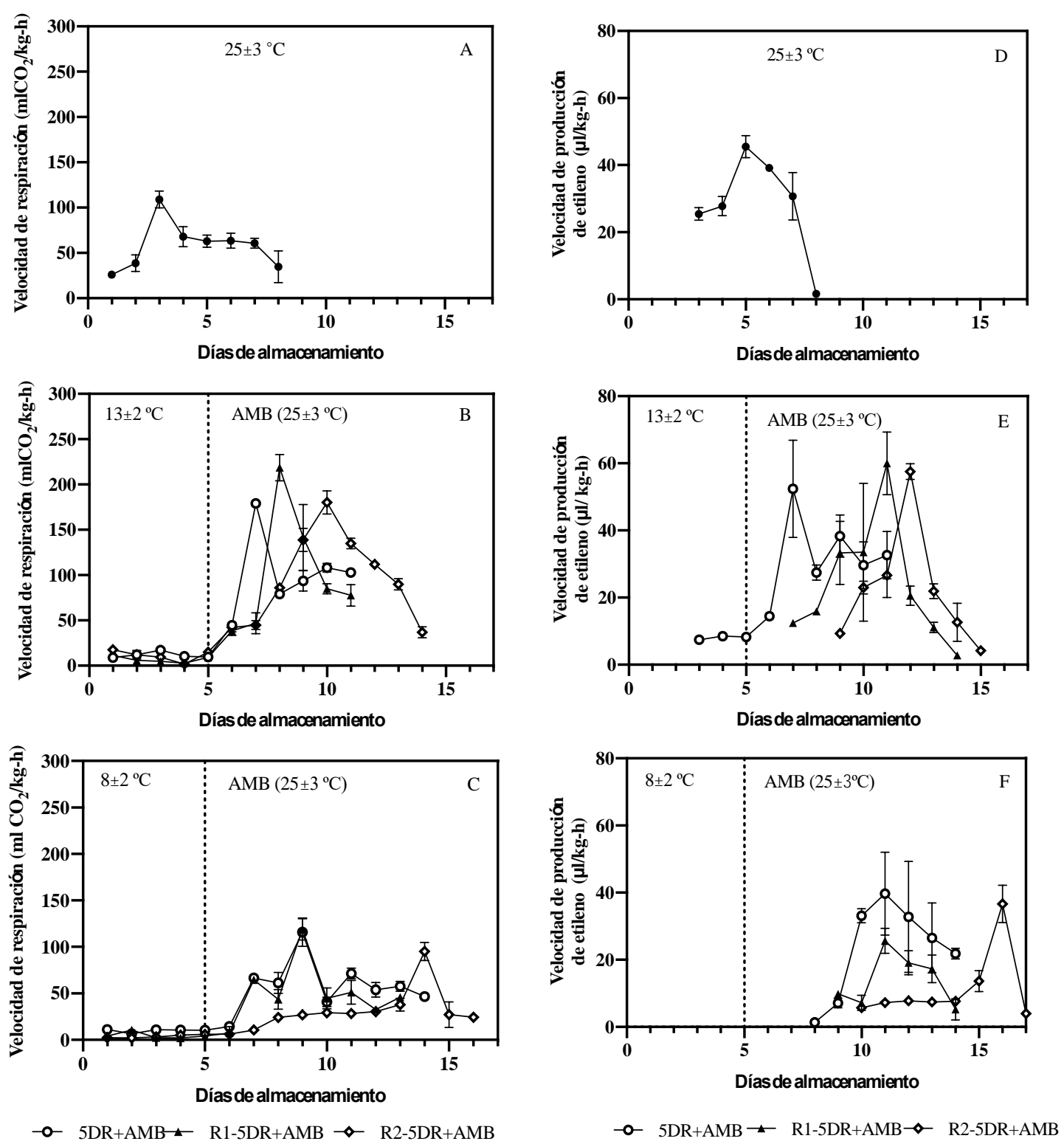

Figura 1. Velocidad de respiración (A, B, C) y velocidad de producción de etileno (D, E, F) en frutos de yaca. Frutos almacenados a temperatura ambiente (AMB, $\left.25 \pm 3^{\circ} \mathrm{C}\right)(\mathrm{A}, \mathrm{D})$, frutos tratados con los recubrimientos ( $\mathrm{R} 1$ y $\mathrm{R2}$ ) y sin recubrimiento con refrigeración por 5 días (5DR) a $13 \pm 2^{\circ} \mathrm{C}(\mathrm{B}, \mathrm{E})$ o $8 \pm 2^{\circ} \mathrm{C}(\mathrm{C}, \mathrm{F})$ y posterior almacenamiento a temperatura ambiente hasta su madurez de consumo. 
Se observó el efecto significativo $(p<0.05)$ de la temperatura de refrigeración por cinco días en la $\mathrm{VR}$, ya que en los frutos almacenados a $8{ }^{\circ} \mathrm{C} 5 \mathrm{D}+\mathrm{AMB}$ la VR fue menor respecto a los frutos que fueron almacenados a $13{ }^{\circ} \mathrm{C} 5 \mathrm{D}+\mathrm{AMB}$. En comparación con los frutos testigo, en los frutos refrigerados la VR máxima se retrasó 4 y 5 días. Mathooko y Tsunashuma (2001) mencionan que las temperaturas de refrigeración disminuyen las actividades enzimáticas involucradas en el complejo proceso de la respiración y las temperaturas mayores aumentan la actividad enzimática, por lo tanto, la VR aumentó cuando los frutos de yaca se almacenaron a temperaturas mayores que las de refrigeración.

La VR de los frutos de yaca fue afectada tanto por la temperatura de refrigeración como por los recubrimientos usados, ya que se observó que el recubrimiento R1 no tuvo efecto significativo, posiblemente por ser poco hidrofóbico de acuerdo con el fabricante; sin embargo, el recubrimiento Semperfresh $^{\mathrm{TM}}$ (R2) disminuyó la VR atribuyéndose a características mayormente hidrofóbicas. Este tipo de recubrimiento pudo crear una modificación de la atmósfera interna, como resultado de la formación de una película semi-permeable en la superficie del fruto que reduce el oxígeno disponible para el tejido vegetal y disminuye la velocidad de respiración (Narsaiah et al., 2015).

En la Figura 1D se observa el comportamiento de la VPE de frutos testigo, encontrándose la máxima VPE en el quinto día de almacenamiento con un valor de $45.48 \mu \mathrm{kg}^{-1} \mathrm{~h}^{-1}$. En los frutos sin recubrimientos almacenados a $13{ }^{\circ} \mathrm{C} 5 \mathrm{D}+\mathrm{AMB}$ (Figura 1E), la aparición de la máxima VPE fue el séptimo día $\left(52.34 \mu 1 \mathrm{~kg}^{-1} \mathrm{~h}^{-1}\right)$, mientras que en los frutos recubiertos con R1 y R2 se observa un pico de etileno para los días $11\left(59.94 \mu \mathrm{kg}^{-1} \mathrm{~h}^{-1}\right)$ y $12\left(57.53 \mu \mathrm{kg}^{-1} \mathrm{~h}^{-1}\right)$ de almacenamiento, respectivamente. Para los frutos sin recubrir y recubiertos con $\mathrm{R} 1$ almacenados a $8{ }^{\circ} \mathrm{C} 5 \mathrm{D}+\mathrm{AMB}$ (Figura 1F), se observa la presencia de etileno una vez que los frutos fueron almacenados a temperatura ambiente y a partir del noveno día, alcanzando la mayor VPE en el onceavo día (39.69 y $25.67 \mu \mathrm{kg}^{-1} \mathrm{~h}^{-1}$, respectivamente), mostrando que la VPE se vio disminuida por efecto del R1; sin embargo, los frutos con R2 tuvieron menor VPE en el onceavo día y presentaron máxima VPE hasta el día 16 de almacenamiento $\left(36.63 \mu \mathrm{kg}^{-1} \mathrm{~h}^{-1}\right)$.

Se observó un efecto significativo $(p<0.05)$ de la temperatura de almacenamiento en la VPE, en los frutos refrigerados a 13 o $8^{\circ} \mathrm{C}$ la VPE fue menor que a $25^{\circ} \mathrm{C}$ durante el tiempo de refrigeración, pero ésta aumentó al salir de refrigeración, siendo mayor a $13{ }^{\circ} \mathrm{C}$. Se ha reportado que el almacenamiento a temperaturas bajas disminuye la producción autocatalítica del etileno debido a que puede disminuir la actividad de la aminociclopropanocarboxilato oxidasa (ACC oxidasa, enzima que interviene en la síntesis del etileno) durante el almacenamiento a esas temperaturas; sin embargo, ésta puede aumentar cuando los frutos son transferidos a condiciones de maduración (Montalvo et al., 2007).

Así mismo, hubo efecto ( $p<0.05$ ) de los recubrimientos en la disminución en la VPE, el recubrimiento R1 tiende a disminuir la concentración del etileno y el recubrimiento R2 disminuyó y retraso la máxima VPE. Esto se atribuye a que los recubrimientos pudieron disminuir la disponibilidad interna de oxígeno, el cual se requiere por la ACC oxidasa para sintetizar etileno (Abeles et al., 1992), aunque el recubrimiento R2 fue mucho más efectivo; lo cual se atribuye a que este recubrimiento disminuye la respiración y la difusión de $\mathrm{CO}_{2} \mathrm{y} \mathrm{O}_{2}$ en los frutos, creando una atmósfera modificada dentro de los tejidos del fruto lo que es efectivo en retrasar el proceso de maduración (Narsaiah et al., 2015). 


\section{Pérdida fisiológica de peso}

Los frutos testigos almacenados a $25^{\circ} \mathrm{C}$ al día 8 tuvieron una pérdida fisiológica de peso (PFP) de 11.9\% (Figura 2A). El almacenamiento a $13{ }^{\circ} \mathrm{C} 5 \mathrm{D}+\mathrm{AMB}$ causó una PFP de $11.38 \%$ (día 11); sin embargo, si se aplican recubrimiento en los frutos, se disminuye el porcentaje de pérdida entre 17 y $20 \%$ para el mismo día de almacenamiento (día 11). A medida que los frutos recubiertos tienen mayor tiempo de almacenamiento a temperatura ambiente para alcanzar su maduración (14 días), la PFP fue ligeramente mayor $12.59 \%$ para frutos con R1 y de $13.06 \%$ para frutos con R2 (Figura 2B). La transpiración de los frutos de yaca se refleja en la pérdida de agua y esto en pérdida fisiológica de peso a medida que avanza la maduración del fruto (Mata-Montes de Oca et al., 2007).

$\mathrm{Al}$ almacenar los frutos a $8{ }^{\circ} \mathrm{C} 5 \mathrm{D}+\mathrm{AMB}$ sin y con recubrimiento (Figura 2C), en el día 11 de almacenamiento la PFP fue de $10.09 \%$ para frutos sin recubrir y de $8.33 \%-8.47 \%$ para frutos recubiertos; es decir, una reducción de la pérdida de peso de 11.33 y $27 \%$, que los frutos almacenados a $13{ }^{\circ} \mathrm{C} 5 \mathrm{D}+\mathrm{AMB}$ (Figura 2B) en comparación con frutos almacenados a $25^{\circ} \mathrm{C}$. La PFP final fue de $13.38 \%$ para frutos sin recubrir, $12.44 \%$ para frutos con R1 a los 14 días de almacenamiento y de $13.21 \%$ para los frutos con $\mathrm{R} 2$ a los 17 días de almacenamiento.

No hubo efecto significativo ( $p>0.5$ ) por tipo de recubrimiento sobre la PFP, pero si fue menor en los frutos sin recubrir. Además, durante la refrigeración la PFP fue menor, pero una vez en condiciones de mercadeo a temperatura ambiente $\left(25^{\circ} \mathrm{C}\right)$, aumenta, aunque es menor en los frutos que estuvieron a $8{ }^{\circ} \mathrm{C}$.
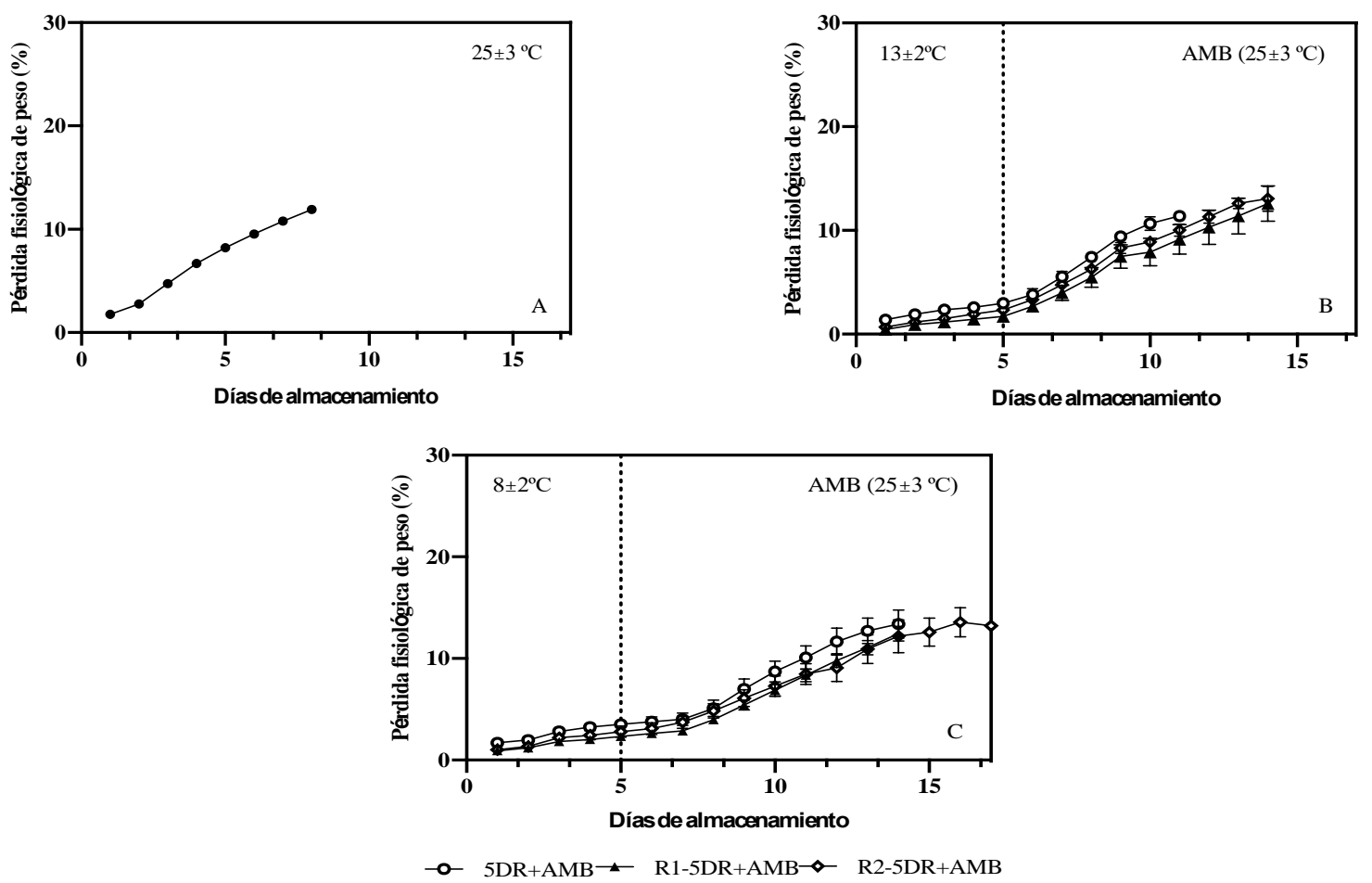

Figura 2. Pérdida fisiológica de peso de frutos de yaca almacenados a temperatura ambiente $(25 \pm 3$ $\left.{ }^{\circ} \mathrm{C}\right)(\mathrm{A})$, frutos sin recubrimiento $\mathrm{y}$ con recubrimiento ( $\mathrm{R} 1 \mathrm{y} \mathrm{R} 2$ ) almacenados en refrigeración por 5 días (5DR) a $13 \pm 2^{\circ} \mathrm{C}(\mathrm{B})$ o $8 \pm 2{ }^{\circ} \mathrm{C}$ (C) y posterior almacenamiento a temperatura ambiente hasta su madurez de consumo. 
La PFP disminuye o aumenta con la temperatura de almacenamiento, lo cual tiene explicación en el metabolismo respiratorio propio de los frutos, además a la ausencia o presencia de un recubrimiento, que actuaría como una barrera que impide el intercambio gaseoso del fruto con el ambiente exterior que lo rodea. La pérdida de agua en los frutos se lleva a cabo por diferencias en la presión de vapor en el medio ambiente y la presión de vapor en el interior de una fruta; sin embargo, también es conocido que la aplicación de recubrimientos ayuda a disminuir la PFP y la efectividad de recubrimiento depende de las características del fruto (tipo de cutícula y velocidad de transpiración), composición y condiciones de almacenamiento (Amarante y Banks, 2001). La aplicación del recubrimiento R2 retrasó en mayor medida la PFP, debido sus características hidrófobas (Saberi et al., 2018). González et al. (2014) reportaron que emulsiones a base de ceras aplicadas a guanábana en postcosecha disminuyeron la pérdida de peso.

\section{Parámetros fisicoquímicos}

La yaca tiene la característica de tener una cáscara gruesa que rodea los bulbos de pulpa, la cual es porosa y de un grosor entre 0.3 a $0.8 \mathrm{~cm}$. El fruto en madurez fisiológica es difícil de penetrar, conforme madura pierde humedad, parece adelgazarse y hacerse flexible, pero sirve como protección a la pulpa de los daños físicos y biológicos externos por mayor tiempo. En la Figura 3 se muestra el comportamiento de la firmeza de los frutos enteros y bulbos de yaca.

La firmeza inicial en todos los frutos fue entre 300 y $350 \mathrm{~N}$, valores semejantes a los reportados por Mata-Montes de Oca et al. (2007) de 330 N, después la firmeza disminuyó. La pérdida de firmeza durante la maduración de frutos climatéricos está relacionada con la acción de enzimas que degradan la pared celular tales como poligalacturonasa, pectinmetilesterasa y $\beta$-galactosidasa entre otras (Montalvo et al., 2009).

En los frutos enteros a $25^{\circ} \mathrm{C}$ la firmeza disminuyó a $239 \mathrm{~N}$ en el día 8 (Figura 3A). Así mismo, en los frutos sin recubrimientos almacenados a 5DR + 3AMB fue de 296.4 y $295 \mathrm{~N}$ respectivamente (Figuras 3B y 3C), hubo efecto significativo $(p<0.05)$ de la temperatura de refrigeración en mantener la firmeza; sin embargo, una vez que los frutos son almacenados a temperatura ambiente en simulación de mercadeo, disminuye rápidamente la firmeza, pero se observa el efecto de la refrigeración, ya que los frutos a $13{ }^{\circ} \mathrm{C} 5 \mathrm{DR}+6 \mathrm{AMB}$ disminuyeron hasta $235 \mathrm{~N}$, mientras que en los frutos almacenados a $8{ }^{\circ} \mathrm{C} 5 \mathrm{DR}+9 \mathrm{AMB}$ alcanzaron una firmeza de $216.7 \mathrm{~N}$; es decir, que los frutos se ablandan de manera similar que frutos almacenados a $25^{\circ} \mathrm{C}$, pero la refrigeración los mantiene más tiempo firmes.

La firmeza de los frutos enteros tratados con los recubrimientos y almacenados a $13{ }^{\circ} \mathrm{C} 5 \mathrm{DR}+$ AMB (Figura 3B) o $8^{\circ} \mathrm{C} 5 \mathrm{DR}+\mathrm{AMB}$ (Figura 3C) no fue estadísticamente diferente $(p>0.05)$, en comparación con los frutos sin recubrir en la mayoría de los días de almacenamiento; solo se observó que en los frutos recubiertos con R2, la firmeza se mantuvo más alta en el último día de almacenamiento. Asimismo, sucedió para los frutos con recubrimiento y almacenados a $8{ }^{\circ} \mathrm{C}$, donde los frutos con aplicación de R2 mantuvieron mayor firmeza, pero sin diferencias significativas. Probablemente no se observó efecto del recubrimiento en la firmeza porque la cáscara del fruto al perder humedad se vuelve flexible y aunque al tacto se siente blanda, al medir la fuerza de ruptura, ésta no es tan baja como se esperaría en la cáscara de un fruto maduro, estos datos coinciden con lo reportado por Mata-Montes de Oca et al. (2007). 

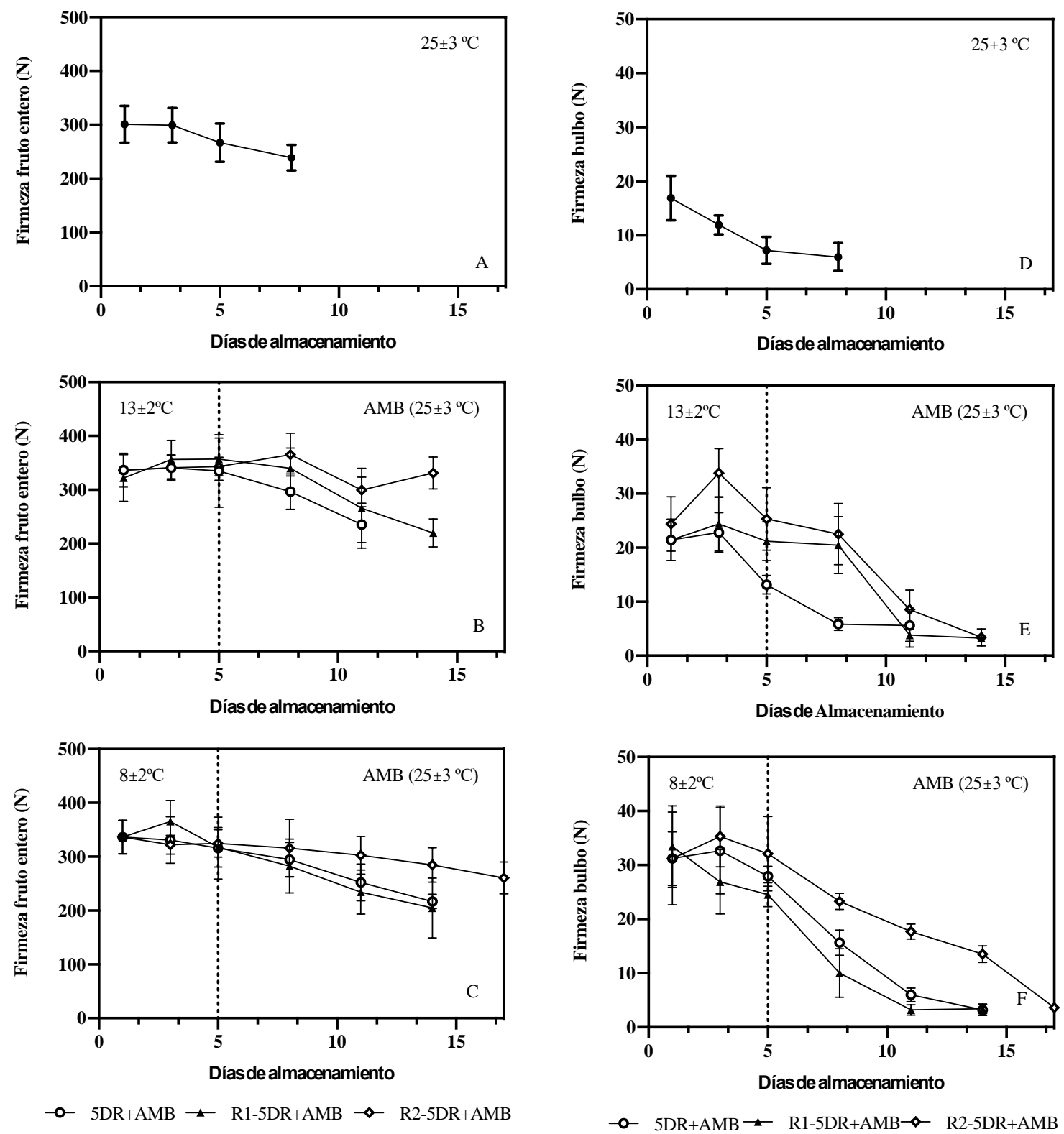

Figura 3. Firmeza externa (A, B, C) e interna (D, E, F) de frutos de yaca. Frutos almacenados a temperatura ambiente $(A, D)$, frutos tratados con recubrimientos $(R 1$ y R2) y sin recubrimiento con refrigeración por 5 días $(5 \mathrm{DR})$ a $13 \pm 2{ }^{\circ} \mathrm{C}(\mathrm{B}, \mathrm{E})$ ó $8 \pm 2{ }^{\circ} \mathrm{C}(\mathrm{C}, \mathrm{F})$ y posterior almacenamiento a temperatura ambiente hasta su madurez de consumo.

Por otra parte, los bulbos de los frutos almacenados a $25^{\circ} \mathrm{C}$ tuvieron una firmeza inicial de $16 \mathrm{~N}$, llegando a valores de $6 \mathrm{~N}$ una vez que los frutos estuvieron maduros (Figura 3D). Los bulbos de los frutos tratados con los recubrimientos y almacenados a $13{ }^{\circ} \mathrm{C} 5 \mathrm{DR}+\mathrm{AMB}$ (Figura 3E) tuvieron mayor firmeza durante el tiempo de refrigeración y los 5 días más en almacenamiento a ambiente en comparación con los bulbos de los frutos sin recubrir a las mismas condiciones de almacenamiento. 
En la Figura 3F, se observa que los bulbos de los frutos con el recubrimiento R1 y almacenados a $8{ }^{\circ} \mathrm{C} 5 \mathrm{DR}+\mathrm{AMB}$ no mostraron diferencia significativa en la firmeza con respecto a los bulbos refrigerados a $8{ }^{\circ} \mathrm{C} 5 \mathrm{DR}+\mathrm{AMB}$ sin recubrir a diferencia de los bulbos de frutos con $\mathrm{R} 2$ que mantuvieron una mayor firmeza durante el tiempo de almacenamiento, alcanzando una firmeza entre los $3 \mathrm{~N}$ hasta el día 17.

La diferencia en el comportamiento de disminución de firmeza entre la cáscara y los bulbos de los frutos cuando maduros, se debe a la composición estructural de cada tejido. Por otra parte, las temperaturas de refrigeración pueden disminuir la actividad de las enzimas que degradan la pared celular como pueden ser celulasas, poligalacturonasas y $\beta$-galactosidasas, sin embargo, la proporción en la disminución enzimática depende de la temperatura de almacenamiento (Jeong et al., 2002; Montalvo et al., 2009).

Es indiscutible que la combinación de la temperatura de refrigeración y el recubrimiento Semperfresh ayudaron a prolongar la vida de anaquel del fruto de yaca hasta 17 días para el caso de refrigeración a $8{ }^{\circ} \mathrm{C}$ por cinco días y posterior simulación de mercadeo. Los resultados encontrados coinciden con lo reportado por Kumar et al. (2018) en frutos de ciruela tratados con Semperfresh, la cual disminuyó la pérdida de firmeza durante el periodo de almacenamiento, por su parte Vargas-Torres et al. (2017), reportaron que los bulbos de yaca con recubrimientos tuvieron mayor firmeza que los frutos sin recubrir.

Coincidió que la VPE fue más baja (Figura 1F) en los frutos recubiertos con R2 y almacenados por 5 días a $8^{\circ} \mathrm{C}$, por lo es posible que la actividad de las enzimas que degradan la pared celular fuera más lenta, tanto por efecto de la temperatura como del recubrimiento (Montalvo et al., 2009). Los SST aumentan durante el proceso de maduración (Cuadros 1 y 2). Inicialmente los frutos cosechados tuvieron entre 7.23 a $8.33{ }^{\circ}$ Brix.

Cuadro 1. Sólidos solubles totales, acidez titulable, $\mathrm{pH}$ y color interno de frutos de yaca almacenados a temperatura ambiente $\left(\mathrm{AMB}, 25 \pm 3{ }^{\circ} \mathrm{C}\right)$, frutos almacenados en refrigeración por 5 días a $13 \pm 2{ }^{\circ} \mathrm{C}$ y después almacenados a temperatura ambiente (5D $\left.13{ }^{\circ} \mathrm{C}+\mathrm{AMB}\right)$, frutos tratados con recubrimientos NaturalShine505-OR ${ }^{\circledR}(\mathrm{R} 1)$ y SemperFresh $^{\circledR}(\mathrm{R} 2)$, almacenados en refrigeración por 5 días a $13 \pm 2^{\circ} \mathrm{C}$ y después almacenados a temperatura ambiente.

\begin{tabular}{ccccc}
\hline $\begin{array}{c}\text { Días de } \\
\text { almacenamiento }\end{array}$ & $25 \pm 3^{\circ} \mathrm{C}$ & $\begin{array}{c}5 \mathrm{DR} 13{ }^{\circ} \mathrm{C} \\
+\mathrm{AMB}\end{array}$ & $\begin{array}{c}\mathrm{R} 1-5 \mathrm{DR} 13{ }^{\circ} \mathrm{C} \\
+\mathrm{AMB}\end{array}$ & $\begin{array}{c}\text { R2-5DR A } 13{ }^{\circ} \mathrm{C} \\
+ \text { AMB }\end{array}$ \\
\hline \multicolumn{4}{r}{ Sólidos solubles totales } & $\left({ }^{\circ}\right.$ Brix $)$ \\
1 & $8.33 \pm 1.53 \mathrm{a}$ & $8.3 \pm 0.14 \mathrm{a}$ & $7.23 \pm 0.26 \mathrm{a}$ & $7.23 \pm 0.26 \mathrm{a}$ \\
5 & $27.05 \pm 0.95 \mathrm{a}$ & $25 \pm 0.23 \mathrm{~b}$ & $13.63 \pm 0.33 \mathrm{c}$ & $13.92 \pm 1.67 \mathrm{c}$ \\
8 & $29.55 \pm 3.52 \mathrm{a}$ & $30.13 \pm 2.41 \mathrm{a}$ & $20.15 \pm 2.09 \mathrm{c}$ & $20.6 \pm 4.8 \mathrm{c}$ \\
11 & & $29.85 \pm 1.12 \mathrm{a}$ & $29.13 \pm 0.9 \mathrm{a}$ & $26.38 \pm 0.57 \mathrm{~b}$ \\
14 & & & $33.48 \pm 0.78$ & $\mathrm{~nm}$ \\
16 & & & & $31.08 \pm 1.49$ \\
\hline
\end{tabular}




\begin{tabular}{|c|c|c|c|c|}
\hline $\begin{array}{c}\text { Días de } \\
\text { almacenamiento }\end{array}$ & $25 \pm 3^{\circ} \mathrm{C}$ & $\begin{array}{c}5 \mathrm{DR} 13^{\circ} \mathrm{C} \\
+\mathrm{AMB} \\
\end{array}$ & $\begin{array}{c}\mathrm{R} 1-5 \mathrm{DR} 13^{\circ} \mathrm{C} \\
+\mathrm{AMB}\end{array}$ & $\begin{array}{c}\mathrm{R} 2-5 \mathrm{DR} \text { A } 13^{\circ} \mathrm{C} \\
+\mathrm{AMB}\end{array}$ \\
\hline \multicolumn{5}{|c|}{ Acidez titulable (\% ácido cítrico) } \\
\hline 1 & $0.17 \pm 0.05 \mathrm{a}$ & $0.14 \pm 0.03 \mathrm{a}$ & $0.18 \pm 0.03 a$ & $0.15 \pm 0.02 \mathrm{a}$ \\
\hline 5 & $0.24 \pm 0.06 \mathrm{a}$ & $0.55 \pm 0.13 b$ & $0.25 \pm 0.04 \mathrm{a}$ & $0.28 \pm 0.01 \mathrm{c}$ \\
\hline 8 & $0.19 \pm 0.02 \mathrm{a}$ & $0.33 \pm 0.04 b$ & $0.37 \pm 0.04 b$ & $0.26 \pm 0.05 b$ \\
\hline 11 & & $0.33 \pm 0.03 \mathrm{a}$ & $0.5 \pm 0.07 \mathrm{~b}$ & $0.32 \pm 0.08 \mathrm{a}$ \\
\hline 14 & & & $0.43 \pm 0.19$ & $\mathrm{~nm}$ \\
\hline 16 & & & & $0.3 \pm 0.01$ \\
\hline \multicolumn{5}{|c|}{$\mathrm{pH}$} \\
\hline 1 & $5.88 \pm 0.4 \mathrm{a}$ & $6.05 \pm 0.1 \mathrm{a}$ & $6.06 \pm 0.13 \mathrm{a}$ & $6.06 \pm 0.13 \mathrm{a}$ \\
\hline 5 & $4.83 \pm 0.05 b$ & $4.46 \pm 0.21 b$ & $5.21 \pm 0.03 \mathrm{a}$ & $4.90 \pm 0.13 a$ \\
\hline 8 & $5.46 \pm 0.28 \mathrm{a}$ & $4.90 \pm 0.08 b$ & $5.05 \pm 0.11 b$ & $4.87 \pm 0.32 b$ \\
\hline 11 & & $5.17 \pm 0.02 b$ & $5.05 \pm 0.12 \mathrm{a}$ & $5.08 \pm 0.07 \mathrm{a}$ \\
\hline 14 & & & $5.29 \pm 0.03$ & $\mathrm{~nm}$ \\
\hline 16 & & & & $5.36 \pm 0.14$ \\
\hline \multicolumn{5}{|c|}{${ }^{\circ}$ Hue $(h)$} \\
\hline 1 & $80.5 \pm 10.73 a$ & $69.1 \pm 1.42 \mathrm{a}$ & $72 \pm 2.32 \mathrm{a}$ & $72 \pm 2.32 \mathrm{a}$ \\
\hline 5 & $64.86 \pm 2.62 \mathrm{a}$ & $70.91 \pm 1.94 b$ & $72.72 \pm 1.68 b$ & $72.54 \pm 3.23 b$ \\
\hline 8 & $64.06 \pm 4.48 \mathrm{a}$ & $68.25 \pm 1.59 \mathrm{a}$ & $72.41 \pm 1.94 b$ & $73.09 \pm 4.46 b$ \\
\hline 11 & & $63.5 \pm 4.17 \mathrm{a}$ & $69.77 \pm 1.99 \mathrm{a}$ & $73.09 \pm 4.46 \mathrm{a}$ \\
\hline 14 & & & $67.86 \pm 2.77$ & $\mathrm{~nm}$ \\
\hline 16 & & & & $69.66 \pm 2.12$ \\
\hline
\end{tabular}

Medias con letras iguales por fila son estadísticamente iguales $(p \leq 0.05)$; $\mathrm{nm}=$ no medido.

Cuadro 2. Sólidos solubles totales, acidez titulable, pH y color interno de frutos de yaca almacenados a temperatura ambiente (AMB, $25 \pm 3{ }^{\circ} \mathrm{C}$ ), frutos almacenados en refrigeración por 5 días a $8 \pm 2{ }^{\circ} \mathrm{C}$ y después almacenados a temperatura ambiente $\left(5 \mathrm{D} 8^{\circ} \mathrm{C}+\mathrm{AMB}\right)$, frutos tratados con recubrimientos NaturalShine505-OR ${ }^{\circledR}$ (R1) y SemperFresh $^{\circledR}(\mathrm{R} 2)$, almacenados en refrigeración por 5 días a $8 \pm 2{ }^{\circ} \mathrm{C}$ y después almacenados a temperatura ambiente.

\begin{tabular}{ccccc}
\hline $\begin{array}{c}\text { Días de } \\
\text { almacenamiento }\end{array}$ & $25 \pm 3^{\circ} \mathrm{C}$ & 5DR $8{ }^{\circ} \mathrm{C}+\mathrm{AMB}$ & $\mathrm{R} 1-5 \mathrm{DR} 8{ }^{\circ} \mathrm{C}+\mathrm{AMB}$ & $\mathrm{R} 2-5 \mathrm{DR} 8{ }^{\circ} \mathrm{C}+\mathrm{AMB}$ \\
\hline \multicolumn{5}{c}{ Sólidos solubles totales $\left({ }^{\circ} \mathrm{Brix}\right)$} \\
1 & $8.33 \pm 1.53 \mathrm{a}$ & $8.33 \pm 1.53 \mathrm{a}$ & $5.9 \pm 0.58 \mathrm{a}$ & $5.90 \pm 0.58 \mathrm{a}$ \\
$5^{*}$ & $27.05 \pm 0.95 \mathrm{a}$ & $13.38 \pm 0.25 \mathrm{~b}$ & $20.38 \pm 4.57 \mathrm{~b}$ & $14.73 \pm 0.78 \mathrm{~b}$ \\
8 & $29.55 \pm 3.52 \mathrm{a}$ & $17.85 \pm 2.48 \mathrm{~b}$ & $23.1 \pm 2.14 \mathrm{c}$ & $16.73 \pm 2.36 \mathrm{c}$ \\
11 & & $28.95 \pm 0.52 \mathrm{a}$ & $29.6 \pm 1.17 \mathrm{a}$ & $20.78 \pm 1.65 \mathrm{a}$ \\
14 & & $26.63 \pm 0.89 \mathrm{a}$ & $28.63 \pm 1.25 \mathrm{a}$ & $\mathrm{nm}$ \\
17 & & & & $29.6 \pm 4.79$ \\
\hline
\end{tabular}




\begin{tabular}{|c|c|c|c|c|}
\hline $\begin{array}{c}\text { Días de } \\
\text { almacenamiento }\end{array}$ & $25 \pm 3^{\circ} \mathrm{C}$ & $5 \mathrm{DR} 8{ }^{\circ} \mathrm{C}+\mathrm{AMB}$ & $\mathrm{R} 1-5 \mathrm{DR} 8{ }^{\circ} \mathrm{C}+\mathrm{AMB}$ & $\mathrm{R} 2-5 \mathrm{DR} 8{ }^{\circ} \mathrm{C}+\mathrm{AMB}$ \\
\hline \multicolumn{5}{|c|}{ Acidez titulable (\% ácido cítrico) } \\
\hline 1 & $0.17 \pm 0.05 \mathrm{a}$ & $0.17 \pm 0.05 \mathrm{a}$ & $0.17 \pm 0.01 \mathrm{a}$ & $0.14 \pm 0.01 \mathrm{a}$ \\
\hline $5^{*}$ & $0.26 \pm 0.07 \mathrm{a}$ & $0.26 \pm 0.07 \mathrm{a}$ & $0.48 \pm 0.08 b$ & $0.31 \pm 0.03 b$ \\
\hline 8 & $0.19 \pm 0.02 \mathrm{a}$ & $0.19 \pm 0.02 \mathrm{a}$ & $0.37 \pm 0.09 b$ & $0.27 \pm 0.04 b$ \\
\hline 11 & & $0.36 \pm 0.06 \mathrm{a}$ & $0.51 \pm 0.11 \mathrm{~b}$ & $0.32 \pm 0.04 \mathrm{a}$ \\
\hline 14 & & $0.24 \pm 0.02 \mathrm{a}$ & $0.4 \pm 0.08 b$ & $\mathrm{~nm}$ \\
\hline 17 & & & & $0.28 \pm 0.06$ \\
\hline \multicolumn{5}{|c|}{$\mathrm{pH}$} \\
\hline 1 & $5.88 \pm 0.4 \mathrm{a}$ & $5.88 \pm 0.14 \mathrm{a}$ & $5.86 \pm 0.15 a$ & $5.86 \pm 0.15 a$ \\
\hline $5^{*}$ & $4.83 \pm 0.05 a$ & $4.89 \pm 0.09 \mathrm{a}$ & $4.66 \pm 0.17 \mathrm{a}$ & $4.89 \pm 0.08 \mathrm{a}$ \\
\hline 8 & $5.46 \pm 0.28 \mathrm{a}$ & $5.53 \pm 0.18 \mathrm{a}$ & $5.06 \pm 0.45 \mathrm{a}$ & $4.91 \pm 0.07 b$ \\
\hline 11 & & $5.01 \pm 0.11 \mathrm{a}$ & $5.29 \pm 0.06 \mathrm{a}$ & $5.1 \pm 0.04 \mathrm{a}$ \\
\hline 14 & & $5.21 \pm 0.01 \mathrm{a}$ & $5.26 \pm 0.04 \mathrm{a}$ & $\mathrm{nm}$ \\
\hline 17 & & & & $5.28 \pm 0.05$ \\
\hline \multicolumn{5}{|c|}{${ }^{\circ}$ Hue $(h)$} \\
\hline 1 & $80.5 \pm 10.73 a$ & $78.6 \pm 11.71 \mathrm{a}$ & $77.73 \pm 3.45 a$ & $77.73 \pm 3.45 a$ \\
\hline $5^{*}$ & $64.86 \pm 2.62 \mathrm{a}$ & $71.17 \pm 2.12 b$ & $70.05 \pm 3.89 b$ & $75.39 \pm 2.19 b$ \\
\hline 8 & $64.06 \pm 4.48 \mathrm{a}$ & $73.75 \pm 3.81 b$ & $75.66 \pm 2.84 b$ & $77.05 \pm 4.16 b$ \\
\hline 11 & & $63.94 \pm 9.54 a$ & $65.07 \pm 2.92 \mathrm{a}$ & $72.94 \pm 1.51 b$ \\
\hline 14 & & $69.96 \pm 5.61 \mathrm{a}$ & $66.31 \pm 0.83 \mathrm{a}$ & $\mathrm{nm}$ \\
\hline 17 & & & & $64.68 \pm 2.51$ \\
\hline
\end{tabular}

Medias con letras iguales por fila son estadísticamente iguales $(p \leq 0.05) .{ }^{*}=$ último día en refrigeración; $\mathrm{nm}=$ no medido.

En los frutos madurados a $25{ }^{\circ} \mathrm{C}$ se alcanzaron $29.6{ }^{\circ}$ Brix al día 8 y en los frutos que fueron refrigerados a $13{ }^{\circ} \mathrm{C} \mathrm{u} 8{ }^{\circ} \mathrm{C} \mathrm{DR}+\mathrm{AMB}$ sin recubrimientos, se alcanzaron valores muy similares, pero al día 11 y 14, respectivamente. Esto muestra el efecto que tiene la temperatura de refrigeración sobre el retardo en el desarrollo de los SST por seis días.

Por otro lado, hubo diferencias significativas $(p<0.05)$ en el contenido de SST entre los frutos con ambos recubrimientos y almacenados a $13{ }^{\circ} \mathrm{C}+\mathrm{AMB}$, ya que los frutos con $\mathrm{R} 1$ desarrollaron el mayor contenido de SST, al día 14 con $33.48^{\circ}$ Brix y los frutos con R2 tuvieron $31.08{ }^{\circ}$ Brix el día 16.

En los frutos refrigerados a $8{ }^{\circ} \mathrm{C}$ con recubrimientos se observó un comportamiento similar en los SST, alcanzando valores entre 26.6 a $28.6^{\circ}$ Brix en los días 11 - 14 los frutos recubiertos con R1, pero en los frutos recubiertos con R2 se extendió su madurez hasta el día 17 (5DR + 12AMB) con $29.6^{\circ}$ Brix. El R2 tuvo mayor efecto en retardar el desarrollo de los sólidos solubles, alcanzando los valores esperados a madurez de consumo 8 o 7 días más que los almacenados a temperatura ambiente 3 y 5 días respecto a los refrigerados sin recubrir y entre 2 y 3 días respecto al recubrimiento $\mathrm{R} 1$. 
Los SST totales aumentan conforme el fruto de yaca madura y se debe a la degradación de los polisacáridos (Vargas-Torres et al., 2017). Los recubrimientos también tienen un efecto importante en este parámetro; Velickova et al. (2013) informaron que, en fresas recubiertas este comportamiento se debe a una metabolización más lenta de los carbohidratos durante el tiempo de almacenamiento.

Las propiedades de barrera del recubrimiento R2 y la temperatura de almacenamiento de $8{ }^{\circ} \mathrm{C}$, disminuyeron la producción de etileno, por lo que el aumento de SST se retrasó más días en los frutos con estos tratamientos. Los SST encontrados en este experimento se encuentran dentro del rango reportado por Shamsudin et al. (2009) de entre 19.03-32.53 ${ }^{\circ}$ Brix en jugo de bulbos de yaca almacenada a temperatura ambiente de $27^{\circ} \mathrm{C}$.

La acidez titulable y pH se muestran en el Cuadro 1 y 2. En todos los bulbos, se observó una ligera tendencia a aumentar de la AT y por consecuencia el pH a disminuir. Vargas-Torres et al. (2017) y Mata-Montes de Oca et al. (2007) reportaron un comportamiento diferente de la AT y pH en bulbos de yaca con recubrimientos, ya que la AT disminuye y el $\mathrm{pH}$ desciende, esto puede ser debido a la diferencia en la materia prima, ya que en este experimento se usó un material vegetal no estudiado anteriormente.

Se observa que solo en los frutos que fueron almacenados a $13{ }^{\circ} \mathrm{C}$ o $8{ }^{\circ} \mathrm{C} \mathrm{DR}+\mathrm{AMB}$ con recubrimientos, se retrasó ligeramente $(p<0.05)$ el cambio en la AT y $\mathrm{pH}$ con respecto a los frutos testigo. Los recubrimientos comestibles tienen propiedades de barrera que reducen la permeabilidad superficial de los frutos al $\mathrm{O}_{2}$ y al $\mathrm{CO}_{2}$, lo que conduce a un aumento de la concentración de $\mathrm{CO}_{2}$ en los tejidos frutales y a una disminución de la concentración de $\mathrm{O}_{2}$ que con lleva a retrasar la maduración (de Wild et al., 2005), esto conlleva a concluir que el retraso en la VR y VPE también retrasan la evolución de la AT y pH en este tratamiento.

Respecto al color, los bulbos de yaca cambiaron de un color amarillo claro a naranja intenso en todos los tratamientos. El tono de color amarillo claro en los bulbos en estado de madurez fisiológica tiene valores de ${ }^{\circ}$ Hue de 72.01-80.5 y disminuye a valores promedio de 63.5-69.66, el cual es el color amarillo-naranja característico en la pulpa de yaca, en todos los bulbos de los frutos evaluados.

El cambio de color de los bulbos fue dependiente de cada tratamiento, los bulbos almacenados a $25{ }^{\circ} \mathrm{C}$ fueron los que cambiaron primero de amarillo claro a naranja seguidos, de los bulbos de frutos almacenados a $13{ }^{\circ} \mathrm{C} 5 \mathrm{DR}+\mathrm{AMB}$ sin recubrir y recubiertos, a $8^{\circ} \mathrm{C} 5 \mathrm{DR}+\mathrm{AMB}$ sin recubrir y recubiertos; por lo tanto, se observaron diferencias significativas $(p<0.05)$ en el desarrollo del color por efecto de la condición de almacenamiento y recubrimiento.

Vargas-Torres et al. (2017) reportaron que el valor de ${ }^{\circ}$ Hue para bulbos de yaca maduros (color amarillo) es 82.52-87.87. Por otro lado, Mata-Montes de Oca et al. (2007) mencionan que el color de los bulbos de yaca es amarillo $\left(92-93^{\circ} \mathrm{Hue}\right)$ en el estado inmaduro y cambia a un color amarillonaranja cuando madura $\left(75^{\circ} \mathrm{Hue}\right)$. Los autores mencionados no reportan que tipo de material vegetal utilizaron. Estos resultados no coinciden con lo que se encontró en este trabajo y esto se debe a que el color de los bulbos de yaca maduros puede variar dependiendo del genotipo. 
El etileno regula la síntesis de enzimas que intervienen en la biosíntesis de carotenoides, si la biosíntesis del etileno se retrasa o inhibe, la síntesis de carotenoides es más lenta o se detiene (Mustilli et al., 1999). Por lo tanto, el efecto de la refrigeración a $8{ }^{\circ} \mathrm{C}$ y aplicación del recubrimiento R2 en el lento desarrollo del color en los bulbos puede estar relacionado con la evolución de la VPE, ya que probablemente hubo una disminución en la actividad de enzimas que sintetizan los carotenoides.

\section{Conclusiones}

Los frutos de yaca del material vegetal 'Agüitada' madura entre los 6-7 días cuando es almacenada a temperatura ambiente. Cuando los frutos de yaca se refrigeran a $13{ }^{\circ} \mathrm{Co} 8{ }^{\circ} \mathrm{C}$ por 5 días y después se maduran a temperatura ambiente, su vida de anaquel es de 9-10 y 11-12, días respectivamente, aunque en los frutos almacenados a $8{ }^{\circ} \mathrm{C}$ por 5 días se observó un ligero daño por frío.

La aplicación de recubrimientos Natural Shine en combinación con almacenamiento en refrigeración por 5 días y posterior condición de mercadeo, no alargó la vida de anaquel de los frutos, aunque se observó una disminución de la pérdida de peso. La aplicación del recubrimiento Semperfresh en combinación con las temperaturas de refrigeración ( 8 y $13{ }^{\circ} \mathrm{C}$, por 5 días) disminuyó la velocidad de respiración, producción de etileno, pérdida de peso y retraso la evolución de los parámetros fisicoquímicos, alargando la vida de anaquel de la yaca entre 13 y 15 días, respectivamente, para su mercadeo.

\section{Agradecimientos}

Los autores(as) agradecen a CONACYT por el financiamiento a través del proyecto SAGARPACONACYT (clave no. 291472).

\section{Literatura citada}

Abeles, F. B.; Morgan, P. W. and Saltveit, M. 1992. Ethylene in plant biology. $2^{\text {nd }}$ (Ed). Academic Press, Inc., San Diego. 414 p.

Amarante, C. and Banks, N. H. 2001. Postharvest physiology and quality of coated fruits and vegetables. In: horticultural reviews, vol. 26. (Ed.). Jules Janick. John Wiley \& Sons, Inc. 161-238 pp.

Antunes, M.; Gago, C.; Cavaco, A. and Miguel, G. 2012. Edible coatings enriched with essential oils and their compounds for fresh and fresh-cut fruit. Recent Pat. Food. Nutr. Agric. 4(2):114-122.

AOAC. 2005. Official Methods of Analysis of The Association of Official Analytical Chemists International. $15^{\text {th }}(\mathrm{Ed})$. Arlington, Virginia. 40-88 p.

De-Wild, H. P. J.; Balk, P. A.; Fernandes, E. C. A. and Peppelenbos, H. W. 2005. The action site of carbon dioxide in relation to inhibition of ethylene production in tomato fruit. Postharvest Biol. Tec. 36(3):273-280.

Espinosa, I.; Ortiz, R.; Tovar, B.; Mata, M. and Montalvo, E. 2013. Physiological and physicochemical behavior of soursop fruits refrigerated with 1-methylcyclopropene. J. Food Quality. 36(1):10-20. 
FAO. 2019. Food and Agriculture Organization on the United Nations. Prevention of post-harvest food losses of fruit, vegetables and root crops. A training manual. 17(2):sp.

González, E. M.; Fernández, A. E. L.; Páez, H. R.; Oca, M. M. M. D. y Gómez, B. T. 2014. Uso combinado de 1-Meticiclopropeno y emulsiones de cera en la conservación de guanábana (Annona muricata). Rev. Brasileira de Fruticultura. 36(1):296-304.

Hernández-Lauzardo, A. N.; Hernández-Martínez, M.; Velázquez-Valle, M. G.; Guerra-Sánchez, M. G. y Melo-Giorgana, G. E. 2007. Actividad antifúngica del quitosano en el control de Rhizopus stolonifer (ehrenb.:fr.) Vuill. y Mucor spp. Rev. Mex. Fitopatol. 25(2):109-113.

Jeong, J.; Huber, D. J. and Sargent, S. A. 2002. Influence of 1-methylcyclopropene (1-MCP) on ripening and cell-wall matrix polysaccharides of avocado (Persea americana) fruit. Postharvest Biol. Technol. 25(3):241-256.

Kumar, P.; Sethi, S.; Sharma, R. R.; Srivastav, M.; Singh, D. and Varghese, E. 2018. Edible coatings influence the cold-storage life and quality of 'Santa Rosa'plum (Prunus salicina Lindell). J. Food Sci. Technol. 55(6):2344-2350.

Love, K. and Paull, E. R. 2011. Jackfruit. Fruits and nuts. College of tropical Agriculture and Human Resources, University of Hawaii at Manoa. F-N-19.

Luna-Esquivel, G.; Alejo-Santiago, G.; Ramírez-Guerrero, L. G.; Arévalo-Galarza, Ma. y DeLourdes, C. La yaca. 2013. (Artocarpus heterophyllus Lam) un fruto de exportación. Agroproductividad. 6(5):65-70.

Mata-Montes, O. M.; Osuna-García, J.; Hernández, E. A.; Ochoa, V. M. y Tovar, G. B. 2007. Efecto del 1-metilciclopropeno (1-MCP) sobre la fisiología y calidad de frutos de Jaca (Artocarpus heterophyllus Lam.). Rev. Chapingo Ser. Hortic. 13(2):165-170.

Mathooko, F. M.; Tsunashima, Y.; Owino, W. Z.; Kubo, Y. and Inaba, A. 2001. Regulation of genes encoding ethylene biosynthetic enzymes in peach (Prunus persica L.) fruit by carbon dioxide and 1-methylcyclopropene. Postharvest Biol. Technol. 21(3):265-281.

Montalvo, E.; Adame, Y.; García, H. S.; Tovar, B. and Mata, M. 2009. Changes of sugars, $\beta$ carotene and firmness of refrigerated Ataulfo mangoes treated with exogenous ethylene. The J. Agric. Sci. 147(2):193-199.

Montalvo, E.; García, H. S.; Tovar, B. and Mata, M. 2007. Application of exogenous ethylene on postharvest ripening of refrigerated 'Ataulfo'mangoes'. LWT-Food Sci. Technol. 40(8):1466-1472.

Mustilli, A. C.; Fenzi, F.; Ciliento, R.; Alfano, F. and Bowler, C. 1999. Phenotype of the tomato high pigment-2 mutant is caused by a mutation in the tomato homolog of DEETIOLATED1. The Plant Cell. 11(2):145-157.

Narsaiah, K.; Wilson, R. A.; Gokul, K.; Mandge, H. M.; Jha, S. N.; Bhadwal, S.; Anurag, R. K.; Malik, R. K. and Vij, S. 2015. Effect of bacteriocin-incorporated alginate coating on shelf-life of minimally processed papaya (Carica papaya L.). Postharvest Biol. Tec. 100:212-218.

Pace International. 2019. Specimen Label Natural Shine ${ }^{\circledR}$ 505-or, Specimen Label Semperfresh ${ }^{\mathrm{TM}}$.

Saberi, B.; Golding, J.; Marques, J.; Pristijono, P.; Chockchaisawasdee, S.; Scarlett, C. and Stathopoulos, C. 2018. Application of biocomposite edible coatings based on pea starch and guar gum on quality, storability and shelf life of 'Valencia' oranges. Postharvest Biol. Technol. 137(2018):9-20.

Saxena, A.; Saxena, T. M.; Raju, P. S. and Bawa, A. S. 2011. Effect of controlled atmosphere storage and chitosan coating on quality of fresh-cut jackfruit bulbs. Food Bioprocess Technology. 6(8):2182-2189. 
Shamsudin, R.; Ling, C. S.; Ling, C. N.; Muda, N. and Hassan, O. 2009. Chemical compositions of the jackfruit juice (Artocarpus heterophyllus L.) Cultivar J33 during Storage. J. Appl. Sci. 9(17):3202-3204.

SIAP. 2019. Servicio de Información Agroalimentaria y Pesquera. Anuario estadístico de la Producción Agrícola 2017.

Teja, T. R.; Santhi, K. K. and Narsingharao, A. 2016. Edible film coating of fresh cut jackfruit. Inter. J. Sci. Environ. Technol. 5(3):1658-1668.

Vargas, T. A.; Becerra L. A.; Sayago, A. S.; Palma, R. H.; Garcia, M. L. and Montalvo, G. E. 2017. Combined effect of the application of 1-MCP and different edible coatings on the fruit quality of jackfruit bulbs (Artocarpus heterophyllus Lam) during cold storage. Sci. Hortic. 214(1):221-227.

Velickova, E.; Winkelhausen, E.; Kuzmanova, S.; Alves, V. D. and Moldão-Martins, M. 2013. Impact of chitosan-beeswax edible coatings on the quality of fresh strawberries (Fragaria ananassa cv Camarosa) under commercial storage conditions. LWT-Food Sci. Technol. 52(2):80-92. 